\title{
Schemas for the analytical estimation of the operational properties of agile systems
}

\author{
Alexander Geyda ${ }^{1, *}$, Igor Lysenko ${ }^{1}$ \\ ${ }^{1}$ Institute for Informatics and Automation of the Russian Academy of Sciences, 14 Line, 39, St. Petersburg, Russia
}

\begin{abstract}
The article states a brief historical sketch of the research conducted on the effectiveness of targeted processes in the operation of systems performed by Russian authors; an overview of some current problems in operational properties research solved outside Russia is made. A conclusion on the prospects of the analytical estimation of the operational properties is provided. The main directions of the research conducted on the operational properties of systems and processes of their operation, such as system potential, operational properties of application of information technologies, are stated. The main features of the research conducted on the operational properties in the view of the current tasks of their research are provided as well as the schemes of analytical estimation of the operational properties applicable to the new tasks are stated that allows for meeting these research challenges using mathematical models.
\end{abstract}

\section{Introduction}

The operational properties of systems and their operation processes (hereinafter referred to as "OP"), as well as the core of the properties defining the quality of systems, processes of their operation and changes, are determined practically (in human activities), as they describe the main aspects of the application of systems in practice. It is better to carry out the improvement of systems and processes of their operation analytically, by solving the mathematical tasks of the OP research. The article describes the main features of the OP study in relation to the current problems being solved by domestic and foreign authors. A conclusion about the prospects of the analytical estimation of OP is made based on the revealed features of the current problems; the schemas of the estimations are also given. These schemas allow for switching to an application of the numerical models of system operation that are necessary for the analytical study of OP.

\section{Peculiarities of the study of operational properties by domestic and foreign authors}

\subsection{Main features of the Russian School of research on system operation effectiveness}

Among the proceedings of Russian scientists in the field of "Investigation of the effectiveness of targeted processes", the work of Academician A. N. Kolmogorov [1] is the first to be described; it states a number of methods for estimating the efficiency of firing. The author proposed the estimation of the effectiveness of firing on the basis of a probability measure. Then, these methods were developed by E. S. Wentzel [2], who worked in the Zhukovsky Air Force Engineering Academy for many years. After the war, she sought to solve the task [3] of estimating the effectiveness of different types of weapons and ammunition and ways of firepower arrangement when shooting at flying objects. According to the academician A. N. Kolmogorov, air defense efficiency was assumed as a probability of hitting an air target, calculated on the basis of the distribution of probable points of a bomb explosion neighboring the target and the conditions of the probabilities of target destruction, depending on the point where the explosion could occur. In the $1970 \mathrm{~s}$, these methods were further developed in the works of authors of the Department of combat effectiveness of the A. F. Mozhaisky Military Space Academy, managed by R. M. Yusupov at that time, who now is the director of the Saint Petersburg Institute for Informatics and Automation of the Russian Academy of Sciences [4, 5]. Moreover, there, at the A. F. Mozhaisky Academy, G. B. Petukhov and his students developed the theory of the effectiveness of targeted processes in their work [6, 7]. They assumed the efficiency as a probabilistic measure of compliance of the analytically calculated characteristics of the random effects of a purposeful process to the targeted (directory specified) values of these characteristics. This measure can be described as the probability of a random vector of effects hitting the target area. Around the same time, some research was conducted to improve the efficiency of processes of engineering, production, and application of various types of military equipment, based on the methods proposed

\footnotetext{
Corresponding author: geida@iias.spb.su
} 
by A. N. Kolmogorov [8-19]. Thus, for example, the research conducted on the effectiveness of missile weapon systems $[8,9]$ were carried out by the team of scientists of the Dzerzhinsky Military Academy of Russian Strategic Missile Forces [11-13] as well. The team of scientists from N. G. Kuznetsov Naval Academy [16] under the leadership of I. A. Ryabinin was awarded the USSR State Prize in the field of science and technologies in 1979 for the design and introduction of methods for high reliability and the effectiveness of weapon systems. In the subsequent years, the effectiveness theory was developed in several directions of research that was conducted in the A. F. Mozhaysky Military Space Academy, as well as at the Department of combat effectiveness of Military Space Forces [14] and the Saint Petersburg Institute of Informatics and Automation of the Russian Academy of Sciences [20-23]. Currently, the development of the effectiveness theory includes researching new operational properties [21] of systems and their operation processes, for example the potential of systems [22] and the efficiency of the application of information technologies [23].

\subsection{Comparison of the Russian School of effectiveness research with the main trends in effectiveness research abroad}

The effectiveness of system operation and other operational properties abroad has been traditionally studied on the basis of economic and econometric methods and models that are closely related to the functioning of the free market, as well as through development of heuristics, i.e. the best practices (according to operational properties), such as PMBOK, CMMI, BABOK, and BPM, especially regarding the application of IT. The description of a number of foreign studies on operational properties and their comparison with domestic results is presented in [21] and [23] (regarding the study of operational properties of IT). It is important to note that due to the differences in models and methods applied, the Russian School of effectiveness study carries out an estimation of operational properties quantitatively, using analytical models of purposeful functioning, based on natural laws. As a result, the prediction on analytical (numerical) models and solving the optimization problems become possible. Meanwhile, foreign scholars have traditionally challenged these tasks with "qualitative", "expert" or "a posteriori" research, while models are rarely analytical. Solutions are gained by the generalization of experience and processing of operational results (a posteriori) using econometric and expert methods. It is obvious that the development of mathematical models for the analytical estimation of operational properties is associated with considerable difficulties. Nevertheless, the need in using analytical models persists. For example, such a need arises when using Decision Management Systems [24], during the control of complex programs [25], application of a new generation IT business analysis [26], and Agile technologies [27], in particular. Analytical estimation could automate the solving of some practical problems that are currently solved with heuristics. The experience accumulated by the domestic school of the effectiveness research can allow for meeting new challenges of current practices based on the analytical estimation of OP, or using both the analytical estimation of OP and the elements of the expert assessment [28]. Let us consider two types of these new, crucial tasks.

\section{Tasks of the study of operational properties of agile systems}

Basic information about the operational properties as properties characterizing the adaptability of systems required for achieving the possible targets during their operation, peculiarities of the estimation of these properties, and the performance of their estimation were introduced in [22]. This information is used as the basis for the study of OP. Reaching the possible targets during operation means getting the possible effects (major results) of the operation, complying with targets, and while under possible conditions. At the same time, the targets and requirements for effects as well as the possibility of obtaining effects are subject to change depending on the operating conditions. The feature of the earlier introduced concepts and principles of OP estimation regarding the improvement of systems and processes of their operation implies, first of all, that such operational properties, as the potential of a system [23], allow for transition actions implemented with changes in system performance, the results of such transition processes, and the results of subsequent operations. Therefore, it becomes possible to estimate OP, taking into account the effects of transition actions, and to solve the mathematical problems mentioned hereinabove. Secondly, the introduced OP allow for switching to the analytical estimation of OP performance, depending on the selected system characteristics, their functioning and improvement (targeted changing), and capability of purpose updating, which allows solving the tasks arising during improvement, such as mathematical problems of analysis and synthesis as well as optimization problems. Examples of operational properties are efficiency, effectiveness, resource intensity, efficiency of system operation, system potential, operational properties of application of information technologies, controllability, and competitiveness [21]. A study of OP includes an evaluation of the OP performance, analysis of OP, and synthesis of system characteristics and its operation, according to the performance of the operational properties. The result of the OP study is a detailed specification of the actions undertaken, i.e. solving the task (mathematical) of action selection. If digital IT is applied, a solution is obtained in the form of a (digital) action model. This model may be used in practice through the targeted changes in practice (focused improvement of activities). The accuracy of OP estimation influences the results (produced using a particular IT) of solving the problems of the selection of actions (transition or fundamental, targeted) followed by the results of the improvement of operating experience. 
The need to investigate the operational properties, solve the problem of choosing actions, and improve practical activities appears from time to time and the processes of its improvement are systematic and can manifest as complex tasks of experience improvement. Thus, for example, the actions planned as a result of solving the problems of OP performance determination can be arranged in the form of a state target program (TP), defined as a set of interrelated activities aimed at addressing the systemic problems of development of the state and society. Such events (actions) can be used to implement changes of different types (control, transition, modernization, innovative). Their planning, preparation, and implementation can cause in their turn (in chain order) the assignment of tasks for the improvement of systems and their components, and improvement of their operation. Such tasks can appear to be newly made, and some additional actions have to be carried out for their addressing. In this regard, a number of following features are to be considered taking into account performance of OP, concepts, and methodology of OP research during improvement of systems and processes of their operation:

Estimation of OP during the improvement of systems requires the predicting of future compliance of effects to the requirements of targeted improvement of systems, their operation and other varying influences. Such improvement of operation and targeted transition actions are associated with possible changes (separate and/or complex) in operation purposes; moreover, the impacts of the environment can lead to changes in the ability to achieve targets (for example, when the given resources are changed). Therefore, it is necessary to investigate the compliance of the effects to the changing requirements of different possible conditions to achieve different targets. For example, this requirement is typical for those tasks of improvement that are nowadays solved with Agile techniques.

All the possibilities of the sequence of transition actions must be kept in mind. Transition actions are purposeful changes from previous to new operation during the operation. Transition actions can cause sequences of repetitive tasks and actions (typical) of improvement and are manifested in the form of the improvement scheme (a loop). It consists of alternating sets of targeted actions of different types, i.e. informative (actions aimed at the assessment of the situation and solving the task of selection of non-informative actions) and non-informative (actions aimed at changing of the system and its operation, as well as targeted actions necessary to obtain final results). Such a loop resembles a control loop with feedback, but it is implemented by a human with the help of engineering means and information technologies. Actions implemented during such sequences are fulfilled in different ways under different conditions because the state of the system changes during the implementation of actions. People are forced to make decisions during the implementation of these actions in order to organize these transition activities and subsequent targeted actions under such changing conditions. For this purpose, certain IT are used (e.g. conventional and/or digital). Each transition action (as one of possible action sequences) can be characterized with separate operational properties. As a result of solving mathematical problems in the research conducted on agile systems and their operation under certain OP performance of certain models of improvement and operation, for example, plans of actions or system projects that are aimed at the achievement of targets under changing environments are obtained.

It should be kept in mind that the need to implement transition actions, characteristics of transition actions, and states after the transition actions that are required for further achievement of targets depends on operational conditions, possible targets, and states in which the functioning system appears before transition actions are implemented. Given the stated conditions, it is required to solve a sequence of tasks of different types (questions that need to be answered) about the ongoing transition and other activities, depending on the observed state and necessary targets. For example, in case it is necessary to obtain an answer to the question, if some actions are required (type of task 1) to reach some other different state (and then, perhaps, an answer to the question of what state and what these actions are), or one can proceed further according to the previous plan of actions, the problem of determining whether the conditions were applied for the continuation of the planned activities arises. If the task of type 1 is solved and shows the result indicating that the actions should be implemented with one of the given alternative methods (for example, according to certain rules), one has to choose a certain method for further actions. Then, a problem (type 2) of the selection of one of the preset modes of actions arises. If the task of type 1 leads to a solution implying the need to move to a new state, requiring the implementation of such actions (from a given set of potential actions), characterized with unknown composition, structure and properties, the following task (type 3) is faced: one should determine the transition actions from a set of allowable actions so that they lead to a desired state. The tasks of the stated types can be combined, and the classification of these tasks can be further continued (for example, for the case when it is necessary to change a set of allowable actions). However, all of these types of tasks are related to the tasks of a choice and need to be solved with any (one or more) IT, irrespective of whether it automated or not.

The loop of changing actions is fulfilled directly or indirectly by someone; this person forms the targets, determines the states and their conformity with targets, predicts (or simulates) the future, updates the tasks of changing operating experience, and then solves the tasks using some IT, and, finally, transforms the practice with or without the help of technical devices.

The study of OP is the basis of implementation of the loop of targeted changes, but such a study can be conducted both on the basis of mathematical methods and models (as in the tasks of the effectiveness theory) as well as heuristically, by the generalization of experiences (as in the above stated examples of the current tasks of improving systems and their operation). The difference between the decisions of the considered 
problems based on an analytical evaluation of OP from the traditional solutions of the problems, based on the application of the above-mentioned Decision Management Systems, as well as from traditional solutions of tasks of integrated program management, from conventional solutions using IT business analysis implies the automation of planning of improvement processes and increase its relevance through the use of analytical models and methods. Specifically, taking into account transition actions during the improvement, formulation and solution of tasks of estimation, analysis, and synthesis of systems and their operation based on numeric, analytically calculated OP performance, allows for proceeding to the formulation and solving of practical problems of improvement of systems and their operation as mathematical tasks of OP research, application of mathematical models, and digital IT for solution of such problems. A typical OP estimation scheme allowing for an analytical evaluation was determined for the analytical estimation of OP performance.

\section{Schemes for operational properties estimation}

\subsection{Types of schemes for operational properties estimation}

Let us introduce the typical schemes for the estimation of operational properties. The first scheme is aimed at the estimation of operation efficiency based on the generalization of the concept of estimating the effectiveness of targeted processes $[6,7]$. It is proposed to name the scheme in honor of the author of the theory of effectiveness of targeted processes in system operation, i.e. the Petukhov scheme. According to an estimation with this scheme, it is assumed that the decision on improving actions during their implementation, improving systems and processes of their operation does not affect (or not accepted for) the functioning, while the actions are aimed at achieving one target and are not interrupted.

The second scheme generalizes the first scheme to account for a plurality of possible operations in order to reach different targets under different conditions. According to this scheme, it is assumed that the possible improvements are determined in advance, actions may be interrupted when the target is changed, and then certain transition improvement actions determined before the start of operation.

Finally, the third scheme summarizes the first two schemes in order to account for both possible transition actions selected before their application to achieve different targets and targeted transition actions selected and implemented during operation, depending on the prevailing conditions.

\subsection{Petukhov's scheme of operational properties estimation}

Let us introduce $I_{p}$ as a value of measure on the set, $p$ as a function defining this measure, $Y$-set of vectors of random characteristics of operational effects (characteristics of operational quality), $R$-set of vectors of the required component-wise relations between random values of effects of characteristics and their desired values, $Y_{r}$-set of vectors of characteristics values of the required functioning effects. Then, the estimation of $I_{p}$ is set by the following scheme:

$$
\begin{aligned}
& p: Y \times R \times Y^{r} \rightarrow[0,1] \\
& I_{p}=p\left(Y \times R \times Y^{r}\right)
\end{aligned}
$$

$I_{p}$ is the measure value of the possibility indicating that the predicate in parentheses takes the value "true" or the value indicating that the random event corresponding to the predicate occurs.

Thus, if $Y=y$ is a random variable defined on the axis of real numbers, $R$ is the ratio "at most", $y^{r}$ is the point at the real axis (the required limit value of the random variable $y$ ), then $p=F_{y}(x), I_{p}=F_{y}\left(y^{r}\right)$ is the value of the function of distribution of the random variable $y$ in the point $y^{r}$.

\subsection{Scheme of operational properties estimation given that the transition processes are known during target changing}

Let us introduce $Y^{r}(t)$ as a random process modeling any possible accidental changes $Y^{r}$ in time (which corresponds to the possible changes in targets of system operation), $t_{0}$ as the start moment of the system operation, $Y^{r}=Y^{r}\left(t_{0}\right), T$ as a target duration of the system operation. Then, the scheme of the estimation of operational properties would be of the following type:

$$
\begin{gathered}
p:\left(Y \times R \times Y^{r}(t), t_{0}, T\right) \rightarrow[0,1] \\
I o p=p\left(Y \times R \times Y^{r}(t)\right), t \in\left[t_{0}, T\right]
\end{gathered}
$$

$I_{o p}$ is the measuring value of the possibility that the predicted values of system operation effects (under varying targets) comply with the desired values of effects in the corresponding way. Whereby:

$$
I_{o p}\left(t_{0}\right)=I_{p}
$$

If the requirements are changed and corresponding changes can be determined before operation, it is necessary to plan a transition process (with the characteristics $u$ ) from one operation to another under the stated changes, which makes it possible to estimate the value of $I_{o p}$. However, if characteristics $\mathrm{u}$ of transition actions cannot be determined in advance and depend upon the state of the system and the environment during operation, it is necessary to use the third estimation scheme. According to this scheme, all transition actions are a sequence of changing actions depending upon the state $\mathrm{s}$ (a state is the set of properties performance at a given time) during the operation of the system and the environment. 


\subsection{Scheme of the estimation of operational properties given the processes of improvement depend on system states during its operation}

The last OP research scheme is used when sequences of transition processes described with the characteristics $u$ are planned and implemented during operation, depending on achieved states of the system and the environment $\mathrm{s}$. Transition actions are a set of actions and relations between them, selected in accordance with different IT applied to change the "target" action and (or) necessary for the better achievement of changed or unchanged targets in view of the state that would result when the target is complete. These actions show the aimed effects because of the actions selected for the "target" process and changing its effects. At the same time, support resources are spent for their implementation. The constraint of $u(s)$ describes the characteristics of transition processes necessary for calculating the effects of transition actions and then targeted actions. Characteristics of the sequence $u c=u_{1 \ldots}$. $u_{n}$ of such transition actions out from the plurality of possible sequences characteristics $U c$ depend on the characteristics of the manifested sequences of states $s c=s_{1} \ldots s_{n}$ out of the plurality of possible sequences of states Sc. The scheme of estimation of operational properties in this case is as follows:

$$
\begin{gathered}
p: Y(U c(S c), t) \times R \times Y r(t) \rightarrow[0,1] \\
I_{o p s}=p\left(Y(U c(S c), t) \times R \times Y^{r}(t), t \in\left[t_{0}, T\right],\right. \\
s c \in S c, u c \in U c
\end{gathered}
$$

$I_{\text {ops }}$ is the measuring value of the possibility that the predicted values of operation system effects (under varying targets and transition actions) meet the required values of effects in a corresponding way, in accordance with these targets. Note that the possible sequences sc and $u c$ depend on applied IT. At this, the previous estimation scheme complies with $U c=u$ :

$$
I_{o p s}(U c(S c), t)=I_{o p s}(u, t)=I_{o p}(t)
$$

\section{Conclusion}

According to a brief analysis of the research conducted on the effectiveness of the targeted processes of system operation and a number of other operational properties performed by domestic and foreign authors, it was concluded on the relevance of solving the current problems of OP research based on the analytical estimation of OP performance. The work describes the main features of such important OP research tasks as the tasks of the research of operational properties of agile systems and the tasks of the research of operational properties when using IT. The work states some schemes of analytical estimation of the operational properties and discloses their features. The obtained results should lead to a solution for the contemporary problems of the operational properties research using mathematical models.
Performed under support of the RFBR grant No. 16-08-00953

\section{References}

1. A.N. Kolmogorov, Collection of articles on the theory of firing. Steklov Mathematical Institute, 12, 7-25 (1945)

2. E.S. Wentsel, Y.M. Likhterev, U.G. Milgram, I.V. Khudyakov, Basics of fighting efficiency and operations research (Zhukovsky Air Force Engineering Academy, Moscow, 1961)

3. G. Averkina, G. Epshtein, I. Grekova, New World, 4 (2008)

4. A.Ya. Ioffe, V.I. Markov, G.B Petukhov, Probabilistic Methods in Applied Cybernetics (Leningrad, 1976)

5. L.M. Morozov, G.B. Petukhov, V.N. Sidorov, Methodological basis of the effectiveness theory (Leningrad, 1979)

6. G.B. Petukhov, Basics of the theory of efficiency of targeted processes. Part 1. Methodology, methods and models (1989)

7. G.B. Petukhov, V.I. Yakunin, Methodological basis of external designing of targeted processes and purposeful systems (AST, Moscow, 2006)

8. Reliability and efficiency in engineering: a guidebook in 10 volumes (Mechanical Engineering, Moscow, 1988)

9. V. Dubrovsky, M. Kovbich, Independent newspaper, (2013)

10. U.I. Kryuchkov, V.I. Kuznetsov, Research on effectiveness of weapons (Dzerzhinsky Military Academy of Russian Strategic Missile Forces, Moscow, 1980)

11. E.B. Volkov, V.Z. Dvorkin, A.I. Prokudin, Engineering bases of efficiency of missile systems (Mechanical Engineering, Moscow, 1989)

12. V.D. Roldugin, Simulation and evaluation of the Strategic Missile Forces fighting (Dzerzhinsky Military Academy of Russian Strategic Missile Forces, Moscow, 2005)

13. Y.V. Kryuchkov, V.I. Kuznetsov, G.N. Okhotnikov, Simulation and evaluation of military operations and weapons of Strategic Missile Forces (Moscow, Dzerzhinsky Military Academy of Russian Strategic Missile Forces, 1986)

14. I.V. Lysenko, Analysis and synthesis of elaborate engineering systems. Part 1: Analysis and synthesis of systems providing readiness of boost and space vehicles for launching (basic theory) (Voenizdat, Moscow, 1995)

15. G.P. Zhukov, S.V. Vikulov, Military and economic analysis and operations research (Voenizdat, Moscow, 1987)

16. I.A. Ryabinin, Yu.M. Parfenov, Reliability, capability and safety of ship's power systems (Naval 
Academy named after the Soviet Union Fleet Admiral N. G. Kuznetsov, St. Petersburg, 1997)

17. B.S. Fleishman, Elements of the theory of potential efficiency of complex systems (Soviet Radio, Moscow, 1971)

18. V.V. Podinovsky, V.D. Nogin, Pareto-optimal solutions for multiobjective problems (Nauka, Moscow, 1982)

19. V.D. Nogin, Decision-making in multi-objective environment: a quantitative approach (FIZMATLIT, Moscow, 2005)

20. I.V. Lysenko, Proceedings of SPIIRAS, 1, 49-64 (2002)

21. A.S. Geyda, Z.F. Ismailova, I.V. Klitny, I.V. Lysenko, Proceedings of SPIIRAS, 35, 136-160 (2014)
22. A.S. Geyda, I.V. Lysenko, Proceedings of SPIIRAS, 10, 63-84 (2009)

23. A.S Geyda, I.V Lysenko, R.M. Yusupov, Proceedings of SPIIRAS, 42, 5-36 (2015)

24. J. Taylor, Decision Management Systems: A Practical Guide to Using Business Rules and Predictive Analytics (IBM Press, 2011)

25. T. Kendrick, How to Manage Complex Programs (Amacom, 2016)

26. Th.W. Dinsmore, Disruptive Analytics: Charting Your Strategy for Next-Generation Business Analytics (Apress, 2016)

27. The Agile Extension to the $B A B O K{ }^{\circledR}$ Guide. International Institute of Business Analysis (2015)

28. I.V. Lysenko, New in public management, 2, 117 130 (2007) 